Check for updates

Cite this: RSC Adv., 2017, 7, 27932

Received 24th March 2017

Accepted 16th May 2017

DOI: 10.1039/c7ra03461j

rsc.li/rsc-advances

\section{Asymmetric passivation of edges: a route to make magnetic graphene nanoribbons $\dagger$}

\author{
Wen-cai Yi, (D) ab Wei Liu, (D) cd Lei Zhao, ${ }^{\text {b }}$ Rashed Islam, ${ }^{\mathrm{b}}$ Mao-sheng Miao*bc \\ and Jing-yao Liu (D)*a
}

Zigzag graphene nanoribbons (ZGNRs) are known to carry interesting properties beyond graphene, such as finite and variable band gaps. More interestingly, the edges of ZGNRs are magnetic due to single occupation of carbon dangling bonds (DB). However, the magnetic moments at two different edge sides couple antiferromagnetically, leading to a zero global moment for ZGNRs. Furthermore, the application of ZGNRs is limited by the high chemical activity of their edges that can be easily oxidized while exposed in air. It has been proposed and intensively studied to protect the edges by passivating them by hydrogenation or adsorption of other molecules such as $\mathrm{CO}_{2}$. In this work, we systematically studied the stability, the structures and the effect of $\mathrm{CO}_{2}$ adsorption at the edges of ZGNRs. Our calculations confirm the experimental observation that the $\mathrm{CO}_{2}$ molecules can be easily absorbed by the ZGNR edges. More interestingly, our calculations show that the asymmetric $\mathrm{CO}_{2}$ adsorption at two edges of ZGNR yields a ferrimagnetic state of ZGNRs that presents a finite global moment. Furthermore, considering the strong bonding between $\mathrm{CO}_{2}$ groups and ZGNRs, we propose that it can be utilized to stitch arrays of ZGNRs together to form new types of 2D materials that inherit the advantageous properties of the nanoribbons, such as finite gaps and novel magnetic properties.

\section{Introduction}

Zigzag graphene nanoribbons (ZGNRs), synthesized in 2008, have attracted much attention as a candidate material for spintronic devices because of their peculiar electronic structures. ${ }^{2-6}$ It is well known that the ground state of ZGNRs is characterized by spin-polarized electronic states at two edges, which couple through the graphene backbone and result in an antiferromagnetic $(\mathrm{AF})$ arrangement of spins on adjacent atomic sites with zero total magnetic moment. ${ }^{7-11}$ In this regard, many works reported the electronic properties and chemical properties of the edge states of ZGNRs while the structure is modified by defects, ${ }^{12-15}$ doping foreign atoms, ${ }^{16-21}$ hydrogenation, ${ }^{22-30}$ oxidation, ${ }^{31}$ adsorption of some gas molecules ${ }^{32-35}$ and external electric field. ${ }^{36-38}$ However, it remains a challenge to produce ferrimagnetic ZGNRs with modification of the structures.

${ }^{a}$ Laboratory of Theoretical and Computational Chemistry, Institute of Theoretical Chemistry, Jilin University, Changchun, 130023, People's Republic of China. E-mail: ljy121@jlu.edu.cn

${ }^{b}$ Department of Chemistry \& Biochemistry, California State University, Northridge, CA, 91330-8262, USA. E-mail: mmiao@csun.edu

'Beijing Computational Science Research Center, Beijing, 100193, P. R. China

${ }^{d}$ Department of Physics and Astronomy, University of California, Irvine, CA, 92697, USA

$\dagger$ Electronic supplementary information (ESI) available: The spin-polarized electron density of different $\mathrm{CO}_{2}$ concentrations and position, snapshots of the final configurations for molecular dynamics simulation of $\mathrm{ZGNR}^{-\mathrm{CO}_{2}-\mathrm{ZGNR}}$ interface. See DOI: $10.1039 / \mathrm{c} 7 \mathrm{ra} 03461 \mathrm{j}$
The pristine ZGNR edges might be oxidized easily when exposed in the air. The unpaired electrons and the dangling bonds (DBs) are very reactive and can be passivated by various gas molecules or functional groups. Recently, an experimental work showed that $\mathrm{CO}_{2}$ can be irreversibly adsorbed on GNRS edges at ambient temperature, ${ }^{36}$ but structures of the passive edges are not determined. Furthermore, it is not clear how the absorption of $\mathrm{CO}_{2}$ molecules modifies the magnetism of ZGNR edges.

Here, we performed a first-principle study based on density functional theory (DFT) to address the structures and the magnetism of $\mathrm{CO}_{2}$ adsorbed on ZGNRs. Our results confirmed that $\mathrm{CO}_{2}$ can be strongly adsorbed on the edges of ZGNRs with large adsorption energies of $-3.75 \mathrm{eV}$, which is larger than the adsorption energies of many other molecules, ${ }^{39,40}$ such as NO $(-2.29 \mathrm{eV}), \mathrm{NO}_{2}(-2.70 \mathrm{eV})$ and $\mathrm{H}_{2} \mathrm{O}(-2.56 \mathrm{eV})$. Furthermore, different $\mathrm{CO}_{2}$ concentrations can affect the magnetism of ZGNRs, and in some cases produce ferromagnetic edge states. Additionally, we proposed that $-\mathrm{CO}_{2}$ - group can work as bridges to connect ZGNRs by forming $\mathrm{C}-\mathrm{C}$ and $\mathrm{C}-\mathrm{O}$ covalent bonds. As a result, a two dimensional ZGNR- $\mathrm{CO}_{2}-\mathrm{ZGNR}$ structure can be formed, which is metallic and magnetic. This two dimensional material based on ZGNR arrays sewed by $-\mathrm{CO}_{2}$ - groups may have good potentials for applications in $2 \mathrm{D}$ electronic devices.

\section{Calculation method}

All the first principles calculations were carried out by density functional theory (DFT) framework as implemented in the 
Vienna ab initio Simulation Package (VASP code). ${ }^{\mathbf{4 1 - 4 3}}$ The Perdew-Burke-Ernzerhof (PBE) exchange-correlation functional within the generalized gradient approximation (GGA) scheme was employed. ${ }^{\mathbf{4 4 , 4 5}}$ The electron-ion interaction is described by projector-augmented-wave (PAW) potentials. ${ }^{46}$ The energy cutoff is set to $500 \mathrm{eV}$, the Brillouin Zones (BZ) are represented by Monkhorst-Pack ${ }^{47}$ special $k$-point meshes of $9 \times 1 \times 1$ for structural optimizations. A vacuum space of $20 \AA$ in the nonperiodic direction was used to remove any interaction between two neighboring images. Structural optimizations were completed until the remnant force on each atom was less than $0.02 \mathrm{eV}^{-1}$. The electron spin-polarization was considered in all calculations. The adsorption energies $\left(E_{\text {ads }}\right)$ of $\mathrm{CO}_{2}$ on ZGNR were defined by the following formula:

$$
E_{\mathrm{ads}}=\frac{E_{\mathrm{CO}_{2}-\mathrm{ZGNR}}-\left(E_{\mathrm{ZGNR}}+E_{\mathrm{CO}_{2}}\right)}{N_{\mathrm{CO}_{2}}},
$$

where $E_{\mathrm{CO}_{2}-\mathrm{ZGNR}}, E_{\mathrm{ZGNR}}, E_{\mathrm{CO}_{2}}$ and $N_{\mathrm{CO}_{2}}$ are the total energies of the $\mathrm{CO}_{2}$ adsorbed ZGNR, the pristine ZGNR, the $\mathrm{CO}_{2}$ molecule and the number of $\mathrm{CO}_{2}$ molecules, respectively. The molecular dynamics (MD) simulation were also performed to evaluate the stability for typical structures. All the simulations ran for 3 ps containing $2000 \mathrm{MD}$ steps at a finite temperature in the NVT ensemble with a large supercell containing about 100 atoms.

\section{Results and discussion}

We use 8-ZGNR (containing 8 arrays of $\mathrm{C}$ ) as the example system to study the adsorption of $-\mathrm{CO}_{2}$. We also tested $n$-ZGNRs $(n=6$, $7,9,10,11,12$ ) and found negligible difference while comparing (a)

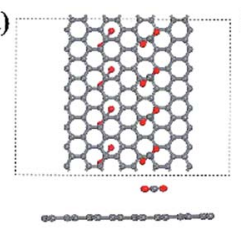

(d)

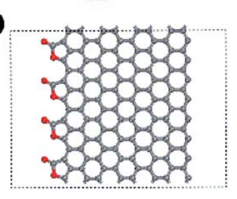

(b)

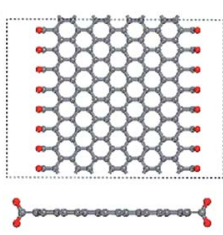

(e)

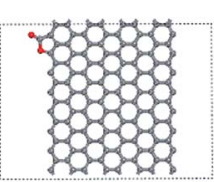

(c)

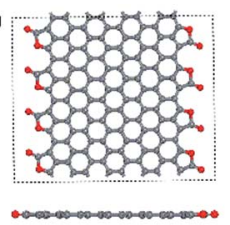

(f)

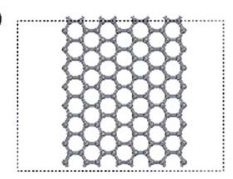

Fig. 1 The structures of 8-ZGNR-t1 (a), 8-ZGNR-s1 (b), 8-ZGNR-8/8 (c), 8-ZGNR-4/8 (d), 8-ZGNR-1/8 (e), 8-ZGNR (f). with 8-ZGNRs results. In order to study different $\mathrm{CO}_{2}$ coverage, 8 repeated unit cells of 8 -ZGNR are included in the model. Several configurations with different adsorption coverages and adsorption sites are constructed and optimized by DFT calculations. These include: (1) on the top of 8-ZGNR (8-ZGNR-t1, Fig. 1a); (2) on both edges of 8 -ZGNR and chemically connected with only carbon atoms in $\mathrm{CO}_{2}$ molecules (8-ZGNR-s1, Fig. 1b); (3) on both edges of 8-ZGNR and connected with both carbon atoms and oxygen atoms in $\mathrm{CO}_{2}$ molecules (8ZGNR-8/8, Fig. 1c); (4) on one edge of 8 -ZGNR with $100 \%$ coverage (8-ZGNR-4/8, Fig. 1d); (5) single $\mathrm{CO}_{2}$ molecule adsorption on one edge of 8 -ZGNR (8-ZGNR-1/8, Fig. 1e). The calculated adsorption energies for all the configurations are listed in Table 1 together with magnetic properties.

For the first adsorption structure 8-ZGNR-t1, the optimization of the structure shows that the distance of the $\mathrm{C}-\mathrm{C}$ bridge connecting $\mathrm{CO}_{2}$ and ZGNR is $3.44 \AA$, indicating a physical adsorption by weak interactions such as van der Waals' force. There is no distinct charge transfer between 8-ZGNR and $\mathrm{CO}_{2}$, and the modification to the electronic structure of ZGNR is minor. So the configuration of 8-ZGNR-t1 remain antiferromagnetic (AFM) after adsorption.

For the second adsorption structure 8-ZGNR-s1, the $\mathrm{C}-\mathrm{C}$ distance of the bridging bond is $1.50 \AA$, which is a bit shorter than the $\mathrm{C}-\mathrm{C}$ bond length of $1.54 \AA$. This indicates chemical adsorption of $\mathrm{CO}_{2}$ molecules on the edges of 8-ZGNR. The adsorption energy is found to be $-0.33 \mathrm{eV}$, showing again the adsorption is chemical. The charges are calculated by the Bader's Quantum Theory of Atoms in Molecules (QTAIM). ${ }^{4-50}$ The results show that there is $0.17 e$ charge transfer from $8-\mathrm{ZGNR}$ to $\mathrm{CO}_{2}$ molecules. The adsorption also alters the structure of $\mathrm{CO}_{2}$ from a linear molecule to a nonlinear bent OCO group. The $\mathrm{O}-\mathrm{C}-\mathrm{O}$ angle becomes $127.40^{\circ}$, and the length of $\mathrm{C}-\mathrm{O}$ changes from $1.18 \AA$ of an isolated $\mathrm{CO}_{2}$ molecule to $1.26 \AA$. The geometry change of $\mathrm{CO}_{2}$ is accompanied by the change of hybridization from $\mathrm{sp}$ to $\mathrm{sp}^{2}$. However, the $\mathrm{sp}^{2}$ hybridization plane of $\mathrm{CO}_{2}$ group is perpendicular to the $\mathrm{sp}^{2}$ hybridization plane of ZGNR.

For the third adsorption structure 8-ZGNR-8/8, different from the structure of 8-ZGNR-s1, both carbon and oxygen atoms of the $\mathrm{CO}_{2}$ molecules bind on two edges of 8-ZGNR, with $\mathrm{C}-\mathrm{O}$ and $\mathrm{C}-\mathrm{C}$ bonds lengths of 1.35 and $1.48 \AA$, respectively, which indicate that 8-ZGNR-8/8 may have a stronger adsorption than 8-ZGNR-s1. The adsorption energy is exceedingly large with a value of $-3.75 \mathrm{eV}$, corresponding to very strong

Table 1 Adsorption energy and energies differences between the FM and AF states (meV per unit) of the studied ZGNRs

\begin{tabular}{|c|c|c|c|c|}
\hline Name & $\begin{array}{l}E_{\text {ads }}(\mathrm{eV} \\
\left.\text { per } \mathrm{CO}_{2}\right)\end{array}$ & $\begin{array}{l}E_{\mathrm{FM}}- \\
E_{\mathrm{AFM}} \text { (meV per unit) }\end{array}$ & $\begin{array}{l}\text { Local magnetic } \\
\text { moment }\left(\mu_{\mathrm{B}} \text { per edge }\right)\end{array}$ & $\begin{array}{l}\text { Total magnetic } \\
\text { moment ( } \mu_{\mathrm{B}} \text { per unit) }\end{array}$ \\
\hline 8-ZGNR-8-t1 & 0.04 & 22.19 & 0.65 & 0.00 \\
\hline 8-ZGNR-8-s1 & -0.33 & -28.48 & 0.48 & 2.00 \\
\hline 8-ZGNR-8/8 & -3.75 & 20.35 & 0.10 & 0.00 \\
\hline 8-ZGNR-4/8 & -3.78 & 22.17 & $0.65(0.1)$ & 8.00 \\
\hline 8-ZGNR-1/8 & -3.90 & 60.03 & $0.65(0.1)$ & 2.00 \\
\hline 8-ZGNR & - & 82.99 & $0.65(0.1)$ & 0.00 \\
\hline
\end{tabular}


(a)

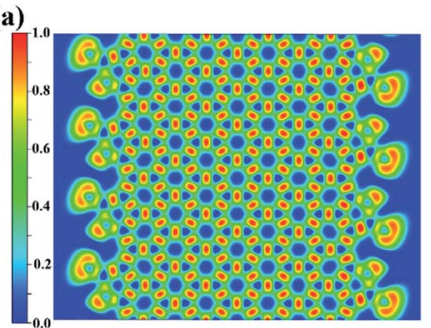

(b)

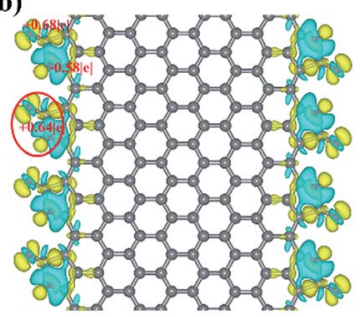

Fig. 2 (a) The calculated ELF of ZGNR-8/8. Green to red indicates the gradually increased charge localization; (b) the charge-density difference for $\mathrm{CO}_{2}$ adsorbed on 8-ZGNR-8/8. Isosurfaces are calculated at 0.004 e $\AA^{-3}$. Yellow was the charge add and the blue was decreased.

chemisorption. The $\mathrm{C}$ atom in $\mathrm{CO}_{2}$ converts to $\mathrm{sp}^{2}$ hybridized and the hybridization plane matches that of ZGNR. Therefore, the $\mathrm{C}$ and $\mathrm{O}$ atoms formed both $\mathrm{s}$ and $\mathrm{p}$ bonds with neighboring $\mathrm{C}$ atoms on ZGNR. The lengths of the $\mathrm{C}-\mathrm{O}$ bonds in $\mathrm{CO}_{2}$ change from $1.18 \AA$ to $1.21 \AA$ and $1.49 \AA$, showing one $\mathrm{C}-\mathrm{O}$ single bond and one $\mathrm{C}=\mathrm{O}$ double bond and the $\mathrm{O}-\mathrm{C}-\mathrm{O}$ angle becomes $118.72^{\circ}$. The nature of their bonding was probed by calculating the electron localization functions (ELF) of this structure. The result is depicted in Fig. 2a. Basins of electron localization could be found between $\mathrm{CO}_{2}$ molecules and 8-ZGNRs, indicating the formation of covalent bonds between them. The QTAIM charge analysis indicates that each carbon atom at the edges of ZGNR loses $0.58 e$ during the adsorption, and the carbon atom in $\mathrm{CO}_{2}$ molecule gains $0.68 e$, and the total charge transfer from 8-ZGNR to one $\mathrm{CO}_{2}$ molecule is $0.64 e$ (Fig. 2b).

These results reveal that $\mathrm{CO}_{2}$ molecules can be strongly adsorbed on the edges of 8-ZGNRs while both $\mathrm{C}$ and $\mathrm{O}$ atoms in $\mathrm{CO}_{2}$ molecule bond with 8-ZGNR. The adsorption energies are larger than $3.0 \mathrm{eV}$, corresponding to very strong chemisorption. Our results confirm the experimental finding that $\mathrm{CO}_{2}$ can be captured by ZGNR if ZGNR grows under the environment of $\mathrm{CO}_{2}$. In this configuration, the carbon and oxygen atoms were both connected with ZGNR whereas the hybridization of carbon changes from $\mathrm{sp}$ to $\mathrm{sp}^{2}$ accompanied by 0.64 electrons transfer from ZGNR to $\mathrm{CO}_{2}$.

The last two configurations represent different $\mathrm{CO}_{2}$ adsorption coverage, based on the coverage, we name them as 8-ZGNR4/8 (Fig. 1d) and 8-ZGNR-1/8 (Fig. 1e). Fig. 1f shows a ZGNR with pristine edges. The adsorption energies and the geometries of the adsorbed $\mathrm{CO}_{2}$ molecules vary only slightly with the $\mathrm{CO}_{2}$ coverage. This is easy to understand since the adsorbed $\mathrm{CO}_{2}$ molecules do not strongly interact with each other. However, as we will show later, the different adsorption coverage has great influence on magnetism of the edges.

To further evaluate the stability of $\mathrm{CO}_{2}$ adsorption system, two typical adsorption structures of 8-ZGNR-8/8 and 8-ZGNR-4/8 were perform MD simulation at $400 \mathrm{~K}$. As shown in Fig. S1, $\dagger$ all those two adsorption structures become stable after 3 ps and neither experience serious disruption nor break, although the atoms shifted along a perpendicular direction, developing a ripple-like structure like other low dimension materials. ${ }^{51}$ The MD simulations demonstrate that the adsorption of $\mathrm{CO}_{2}$ does not break the stability of ZGNRs at ambient temperature.

In order to examine the effect of $\mathrm{CO}_{2}$ adsorption to magnetic order, we calculated the energies of three spin configurations, including non-magnetic (NM), ferromagnetic (FM) and antiferromagnetic (AFM) states. The AFM state consists of local moments oriented oppositely at two edge sides. The energy differences between the FM and AFM states, $\Delta E=E_{\mathrm{FM}}-E_{\mathrm{AFM}}$, of all adsorption models were calculated and listed in Table 1. Negative $\Delta E$ values mean that FM coupling is energetically more favorable, whereas positive values correspond to AFM coupling. All $\Delta E$ values are positive except 8-ZGNR-8-s1, which indicates that the feature of the antiferromagnetic coupling remains after adsorbing $\mathrm{CO}_{2}$ molecules.

The spin densities of the pristine ZGNR and three $\mathrm{CO}_{2}$ adsorbed ZGNR are plotted in Fig. 3. It is well known that zigzag edges ZGNRs are semiconductors with spin-polarized edge states that are ferromagnetically ordered at each edge and antiferromagnetically coupled between two edges (Fig. 3a). Furthermore, the calculations show that the magnetic ordering of the ZGNR edges fully covered by $\mathrm{CO}_{2}$ adsorption (8-ZGNR-8/8) is the same as that of a pristine ZGNR (Fig. $3 \mathrm{~b}$ ). The major difference is that the local magnetic moments are significantly reduced in passivated edges (Table 1). This results agree well with the previous calculation. ${ }^{31}$ More interestingly, while the $\mathrm{CO}_{2}$ coverage is asymmetric between the two sides, there will be net magnetic moments in the ZGNR because the adsorption of $\mathrm{CO}_{2}$ will largely reduce the local moment. If only one edge is covered by $\mathrm{CO}_{2}$ molecules (Fig. 4c), the total magnetic moment will be $8.00 \mu_{\mathrm{B}}$ per cell as used in calculations ( 8 repeating ZGNR units). As a matter of fact, a single $\mathrm{CO}_{2}$ adsorption at one side will contribute $2.00 \mu_{\mathrm{B}}$ for the total magnetic moment (Fig. 4d). We also tested the variation of magnetic moments with different adsorption positions of $\mathrm{CO}_{2}$ molecules (Fig. $\mathrm{S} 2 \dagger$ ) and found that the total magnetism moment is only determined by different number of $\mathrm{CO}_{2}$ molecules adsorbed on two sides. (a)

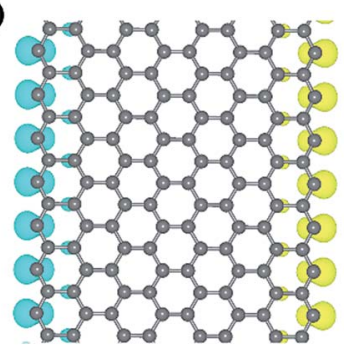

(c)

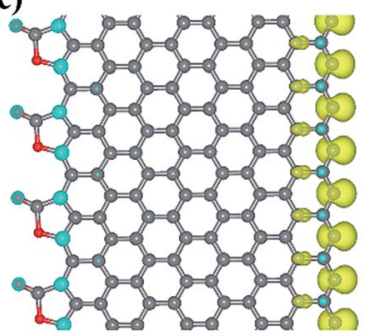

(b)

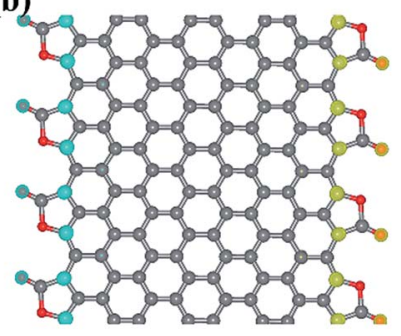

(d)

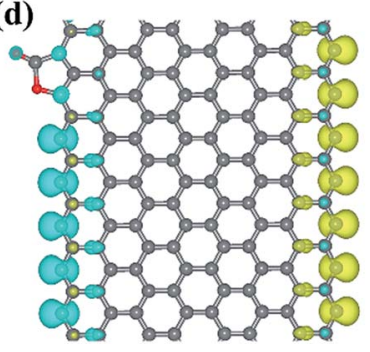

Fig. 3 The spin-polarized electron density of ZGNR (a), ZGNR-8/8 (b), ZGNR-4/8 (c), ZGNR-1/8 (d). Blue was spin up and yellow was spin down. The isosurface value is set to $1.00 \times 10^{-2} \AA^{-3}$. 
(a)

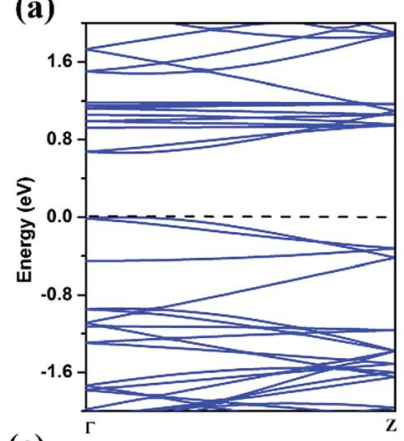

(c)

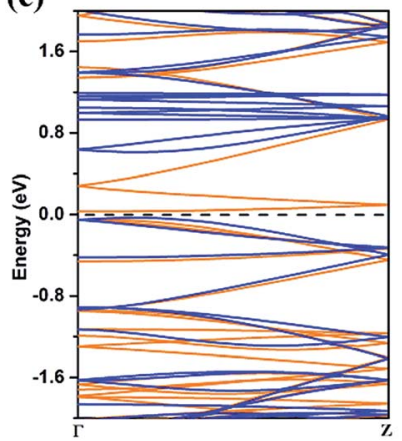

(b)

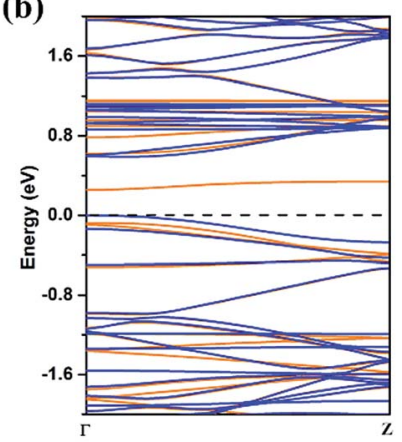

(d)

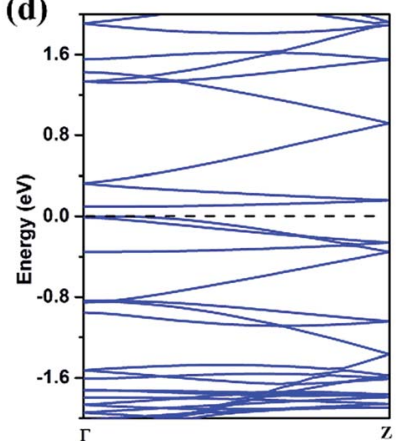

Fig. 4 The band structures of 8-ZGNR (a), 8-ZGNR-1/8 (b), 8-ZGNR4/8 (c), 8-ZGNR-8/8 (d). In (b) and (c), blue and orange lines represent majority spin (down) and minority spin (up) bands.

Therefore, the ZGNR asymmetrically adsorbed $\mathrm{CO}_{2}$ molecules at two edges are ferrimagnetic, since the local moments at two edge sides are antiferromagnetically coupled and yet the system exhibit finite moment.

The band structure of ZGNR is also greatly influenced by the adsorption of $\mathrm{CO}_{2}$. Using PBE functional, we calculated the band structures of ZGNR with different adsorption coverage of $\mathrm{CO}_{2}$ molecules. The results are plotted in Fig. 4. As shown in Fig. $4 \mathrm{a}$, the bare 8-ZGNR has a direct band gap of $0.68 \mathrm{eV}$ at $\Gamma$ point. Considering the use of semi-local exchange-correlation functional (PBE), the band gap might be greatly underestimated in our work. With the adsorption of $\mathrm{CO}_{2}$ molecules, there are several features in the change of the band structures. For the 8ZGNR-1/8 and 8-ZGNR-4/8 coverage, the band structures were different between spin-up and spin-down because the system is magnetic. The 8 -ZGNR-1/8 can be considered as introducing an impurity at the edge. Its effect to the band structure is the inducing of impurity states in the gap (Fig. 4b). As shown in the figure, this impurity state is a deep acceptor state. For 8-ZGNR4/8, a full spin polarized band structure has developed (Fig. 4c). The majority-spin bands exhibit similar band structures as the pristine ZGNR (Fig. 4a); however, the minority-spin bands contain features originated from the absorption. Overall, the material is still semiconducting with a very small gap. Therefore, the ZGNR with $\mathrm{CO}_{2}$ passivation at one side is a low-gap magnetic semiconductor and may find its usage in future twodimensional spintronic devices. 8-ZGNR-8/8 is non-magnetic with overlapping majority and minority bands. While comparing the band structures with the pristine ZGNR, we find

that the original conduction bands are pushed upward for about $0.6 \mathrm{eV}$ which is a result of the passivation of the dangling bonds. A few more bands are introduced in the gap, resembling same features as the minority-spin bands in 8-ZGNR-4/8. Overall, the material is a low-gap semiconductor, the values of band gap were showed in Table S1. $\dagger$

We notice that the $\mathrm{CO}_{2}$ groups can be used to connect two neighbor ZGNR by forming $\mathrm{C}-\mathrm{C}$ and $\mathrm{C}-\mathrm{O}$ bonds. Again, we use 8-ZGNR as example nanoribbons to demonstrate this idea. As shown in Fig. 5a, we constructed a model $\mathrm{CO}_{2}$-stitched ZGNR arrays and optimized its structure using DFT method with PBE functional. The structure belongs to Pmm2 symmetry group. The most stable interface structure consists of $\mathrm{CO}_{2}$ groups bonding to one ZGNR edge with two $\mathrm{C}-\mathrm{O}$ bonds and to another ZGNR edge with one $\mathrm{C}-\mathrm{C}$ bond. The ELF calculation shows strong covalent features for the interface $\mathrm{C}-\mathrm{C}$ and $\mathrm{C}-\mathrm{O}$ bonds. The MD simulations demonstrate this stitched 2D ZGNR arrays are stable up to $700 \mathrm{~K}$. Fig. $\mathrm{S} 3 \uparrow$ shows the structures and fluctuations in the temperature as a function of the simulation time at $300 \mathrm{~K}$ and $700 \mathrm{~K}$, respectively.

Although most dangle bonds at the edges of ZGNR arrays were passivated by $\mathrm{CO}_{2}$ molecules, some of them remain as shown in Fig. 5a. There are single electrons occupying these DBs and may exhibit large local magnetic moments. As a matter of fact, the spin-polarized calculations show that the $\mathrm{CO}_{2}{ }^{-}$ stitched ZGNR arrays are magnetic with a net moment of $1.52 \mu_{\mathrm{B}}$ per unit. Further calculations indicate the energy of ferromagnetic state is $0.57 \mathrm{eV}$ lower than that of non-magnetic state, and is $0.84 \mathrm{meV}$ lower than the antiferromagnetic state. The spin polarized electron density is plotted in Fig. $5 c$ that shows the magnetism moment mainly come from the edge $\mathrm{C}$ atoms containing DB. The local magnetic moments as calculated by the charge density inside the PAW radii is $0.58 \mu_{\mathrm{B}}$ of the carbon atom with DBs.

The band structure calculation indicates the $\mathrm{CO}_{2}$ stitched ZGNR is metallic with impurity states occupied in the middle of band gap and the Fermi level across those states. The further
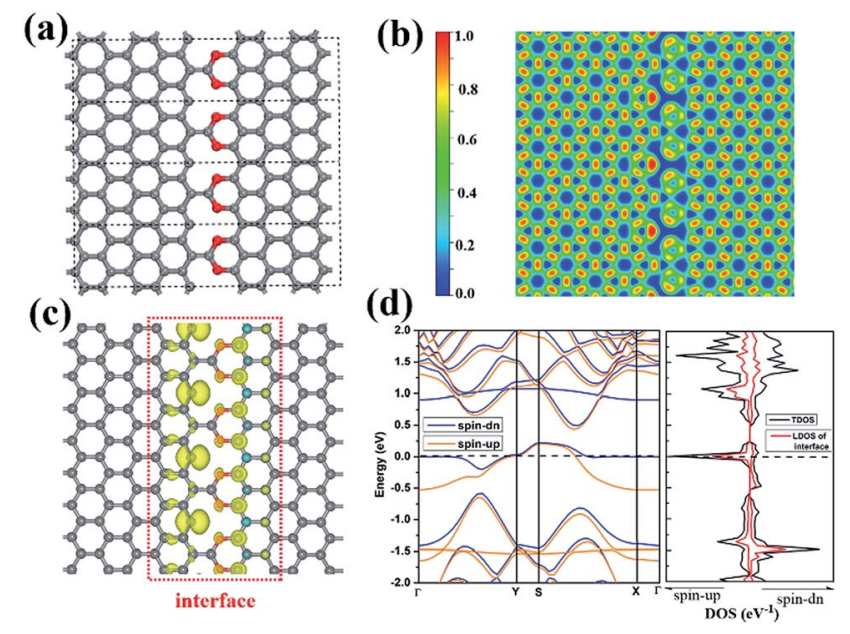

Fig. 5 The structure (a), the ELF (b), the spin-polarized electron density (c), the band structure (d) of $\mathrm{CO}_{2}$ stitched ZGNR. 
analysis by LDOS indicates that most of those states come from the interface between $\mathrm{CO}_{2}$ and ZGNR (Fig. 5d).

\section{Conclusions}

In summary, we have performed first-principles calculations to study the stability, the structures of $\mathrm{CO}_{2}$ molecule adsorption at the edges of ZGNR. Our calculations reveal large adsorption energies, agreeing to the experimental observation of easy adsorption of $\mathrm{CO}_{2}$ molecules at ZGNR edges. The adsorption of $\mathrm{CO}_{2}$ molecules can largely modify the electronic structure of ZGNR. It reduces the overall gap by inducing new edge states in the gap of ZGNR. More interestingly, the adsorption of $\mathrm{CO}_{2}$ at the edges can greatly alter the magnetic properties of ZGNR. Especially, while the adsorption is asymmetric, the ZGNR become ferrimagnetic with large global moment, indicating the fabrication of interesting carbon based low dimensional magnetic materials. Moreover, we propose that the $\mathrm{CO}_{2}$ groups can be used to stitch the ZGNR arrays together to form a new type of 2D materials. The electronic structure calculations show that such materials are ferromagnetic semiconductor with low band gaps.

\section{Acknowledgements}

This work was supported by the National Natural Science Foundation of China (Grants 21373098). The authors are grateful to the referees for their valuable comments, which have significantly improved the manuscript. Part of the calculations are performed on NSF-funded XSEDE resources (TGDMR130005) especially on the Stampede cluster run by Texas Advanced Computing Center.

\section{Notes and references}

1 S. Aigner, L. D. Pietra, Y. Japha, O. Entin-Wohlman, T. David, R. Salem, R. Folman and J. Schmiedmayer, Science, 2008, 319, 1226-1229.

2 V. Barone, O. Hod and G. E. Scuseria, Nano Lett., 2006, 6, 2748-2754.

3 D. E. Jiang, B. G. Sumpter and S. Dai, J. Chem. Phys., 2007, 126, 134701.

4 Q. Yan, B. Huang, J. Yu, F. Zheng, J. Zang, J. Wu, B. L. Gu, F. Liu and W. Duan, Nano Lett., 2007, 7, 1469-1473.

5 F. Wu, E. Kan, H. Xiang, S.-H. Wei, M.-H. Whangbo and J. Yang, Appl. Phys. Lett., 2009, 94, 223105.

6 W. Y. Kim and K. S. Kim, Nat. Nanotechnol., 2008, 3, 408-412. 7 M. Fujita, K. Wakabayashi, K. Nakada and K. Kusakabe, J. Phys. Soc. Jpn., 1996, 65, 1920-1923.

8 S. Okada and A. Oshiyama, Phys. Rev. Lett., 2001, 87, 146803. 9 H. Lee, Y.-W. Son, N. Park, S. Han and J. Yu, Phys. Rev. B: Condens. Matter Mater. Phys., 2005, 72, 174431.

10 C. Tao, L. Jiao, O. V. Yazyev, Y.-C. Chen, J. Feng, X. Zhang, R. B. Capaz, J. M. Tour, A. Zettl, S. G. Louie, H. Dai and M. F. Crommie, Nat. Phys., 2011, 7, 616-620.
11 G. Z. Magda, X. Jin, I. Hagymasi, P. Vancso, Z. Osvath, P. Nemes-Incze, C. Hwang, L. P. Biro and L. Tapaszto, Nature, 2014, 514, 608-611.

12 D. W. Boukhvalov and M. I. Katsnelson, Nano Lett., 2008, 8, 4373-4379.

13 M. Topsakal, E. Aktürk, H. Sevinçli and S. Ciraci, Phys. Rev. B: Condens. Matter Mater. Phys., 2008, 78, 235435.

14 G.-D. Lee, C. Z. Wang, E. Yoon, N.-M. Hwang and K. M. Ho, Phys. Rev. B: Condens. Matter Mater. Phys., 2010, 81, 195419.

15 J. N. B. Rodrigues, P. A. D. Gonçalves, N. F. G. Rodrigues, R. M. Ribeiro, J. M. B. Lopes dos Santos and N. M. R. Peres, Phys. Rev. B: Condens. Matter Mater. Phys., 2011, 84, 155435.

16 T. B. Martins, R. H. Miwa, A. J. da Silva and A. Fazzio, Phys. Rev. Lett., 2007, 98, 196803.

17 S. Dutta and S. K. Pati, J. Phys. Chem. B, 2008, 112, 13331335.

18 S. S. Yu, W. T. Zheng, Q. B. Wen and Q. Jiang, Carbon, 2008, 46, 537-543.

19 S. Dutta, A. K. Manna and S. K. Pati, Phys. Rev. Lett., 2009, 102, 096601.

20 Y. Li, Z. Zhou, P. Shen and Z. Chen, ACS Nano, 2009, 3, 19521958.

21 Z. L. Yu, D. Wang, Z. Zhu and Z. H. Zhang, Phys. Chem. Chem. Phys., 2015, 17, 24020-24028.

22 Y. W. Son, M. L. Cohen and S. G. Louie, Phys. Rev. Lett., 2006, 97, 216803.

23 K. N. Kudin, ACS Nano, 2008, 2, 516-522.

24 T. Wassmann, A. P. Seitsonen, A. M. Saitta, M. Lazzeri and F. Mauri, Phys. Rev. Lett., 2008, 101, 096402.

25 G. Lee and K. Cho, Phys. Rev. B: Condens. Matter Mater. Phys., 2009, 79, 165440.

26 S. Bhandary, O. Eriksson, B. Sanyal and M. I. Katsnelson, Phys. Rev. B: Condens. Matter Mater. Phys., 2010, 82, 165405.

27 R. Yang, Z. Shi, L. Zhang, D. Shi and G. Zhang, Nano Lett., 2011, 11, 4083-4088.

28 J. Zeng, K.-Q. Chen, J. He, X.-J. Zhang and C. Q. Sun, J. Phys. Chem. C, 2011, 115, 25072-25076.

29 X. Q. Deng, Z. H. Zhang, G. P. Tang, Z. Q. Fan and C. H. Yang, Carbon, 2014, 66, 646-653.

30 X. H. Zheng, X. L. Wang, L. F. Huang, H. Hao, J. Lan and Z. Zeng, Phys. Rev. B: Condens. Matter Mater. Phys., 2012, 86, 081408 .

31 O. Hod, V. Barone, J. E. Peralta and G. E. Scuseria, Nano Lett., 2007, 7, 2295-2299.

32 E. J. Kan, Z. Li, J. Yang and J. G. Hou, J. Am. Chem. Soc., 2008, 130, 4224-4225.

33 A. P. Seitsonen, A. M. Saitta, T. Wassmann, M. Lazzeri and F. Mauri, Phys. Rev. B: Condens. Matter Mater. Phys., 2010, 82, 115425.

34 M. Asai, T. Ohba, T. Iwanaga, H. Kanoh, M. Endo, J. CamposDelgado, M. Terrones, K. Nakai and K. Kaneko, J. Am. Chem. Soc., 2011, 133, 14880-14883.

35 E. Akbari, M. T. Ahmadi, M. J. Kiani, H. K. Feizabadi, M. Rahmani and M. Khalid, J. Comput. Theor. Nanosci, 2013, 10, 1301-1304. 
36 E.-J. Kan, Z. Li, J. Yang and J. G. Hou, Appl. Phys. Lett., 2007, 91, 243116.

37 Y. W. Son, M. L. Cohen and S. G. Louie, Nature, 2006, 444, 347-349.

38 S. D. Dalosto and Z. H. Levine, J. Phys. Chem. C, 2008, 112, 8196-8199.

39 B. Huang, Z. Li, Z. Liu, G. Zhou, S. Hao, J. Wu, B.-L. Gu and W. Duan, J. Phys. Chem. C, 2008, 112, 13442-13446.

40 M. Vanin, J. Gath, K. S. Thygesen and K. W. Jacobsen, Phys. Rev. B: Condens. Matter Mater. Phys., 2010, 82, 195411.

41 G. Kresse and J. Hafner, Phys. Rev. B: Condens. Matter Mater. Phys., 1994, 49, 14251-14269.

42 G. Kresse and J. Furthmüller, Comput. Mater. Sci., 1996, 6, 15-50.

43 G. Kresse and J. Furthmüller, Phys. Rev. B: Condens. Matter Mater. Phys., 1996, 54, 11169-11186.
44 J. P. Perdew, K. Burke and M. Ernzerhof, Phys. Rev. Lett., 1996, 77, 3865-3868.

45 P. E. Blöchl, Phys. Rev. B: Condens. Matter Mater. Phys., 1994, 50, 17953-17979.

46 G. Kresse and D. Joubert, Phys. Rev. B: Condens. Matter Mater. Phys., 1999, 59, 1758-1775.

47 H. J. Monkhorst and J. D. Pack, Phys. Rev. B: Condens. Matter Mater. Phys., 1976, 13, 5188-5192.

48 G. Henkelman, A. Arnaldsson and H. Jónsson, Comput. Mater. Sci., 2006, 36, 354-360.

49 E. Sanville, S. D. Kenny, R. Smith and G. Henkelman, J. Comput. Chem., 2007, 28, 899-908.

50 W. Tang, E. Sanville and G. Henkelman, J. Phys.: Condens. Matter, 2009, 21, 084204.

51 W. Liu, M. Miao and J.-y. Liu, J. Mater. Chem. C, 2015, 3, 9921-9927. 